\title{
A Theoretical Study of Convergence Characteristics of a Multiple Channel ANC System
}

\author{
Guoyue Chen ${ }^{\dagger}$ \\ Faculty of Systems Science and Technology, Akita Prefectural University, \\ 84-4 Tsuchiya-Ebinokuchi, Honjo, Akita, 015-0055 Japan \\ Kenji Muto \\ Department of Electronic Engineering, Tokyo Metropolitan College of Aeronautical Engineering, \\ 8-52-1 Minamisenju, Arakawa-ku, Tokyo, 116-8523, Japan
}

(Received 22 March 2004; accepted 20 September 2004)

\begin{abstract}
Most active noise control (ANC) systems are based on feedforward structure with adaptive filters, which are updated with the filtered-x LMS algorithm or the multiple error filtered-x (MEFX) LMS algorithm. The convergence characteristics of these algorithms have been studied mostly in the time domain, and it was found that the convergence properties are subject to the distribution of the eigenvalues of the autocorrelation matrix of the filtered reference signal. Analysis in the time domain, however, requires a great deal of computation, and its physical meaning is unclear. This paper presents a method for evaluating the adaptive algorithm for the ANC system with multiple noise sources and multiple control points in the frequency domain. In this method, the convergence characteristics of the MEFX LMS algorithm are evaluated separately by the determinant or eigenvalues of the matrix, which are composed of transfer functions between the secondary sources and the control points, and the correlations among the outputs of the reference sensors.
\end{abstract}

${ }^{\dagger}$ Member of the International Institute of Acoustics and Vibration (IIAV)

\section{INTRODUCTION}

Multiple channel active noise control (ANC) technology is applied to three-dimensional fields by minimising the sum of the mean square error of all error sensor outputs simultaneously. ${ }^{1-8}$ One of the most widely used adaptive algorithms for the feedforward multiple channel ANC systems is the multiple error filtered-x (MEFX) LMS algorithm, ${ }^{7-9}$ which operates in the time domain and is an extension of the filtered-x LMS algorithm. ${ }^{10-13}$

The convergence characteristics of the MEFX LMS algorithm are subject to the distributions of eigenvalues of the autocorrelation matrix, which consists of a filtered reference signal. ${ }^{14}$ However, since the size of the autocorrelation matrix is very large, analysis in the time domain requires a great computational load. Furthermore, in time domain analysis the physical meaning of the spread in the eigenvalues spreading is unclear. Thus, it is difficult to understand clearly the effect of the input signals and the impulse response of the secondary path on the convergence properties of the MEFX LMS algorithm.

The convergence properties of the filtered-x algorithm can be approximately analysed in the frequency domain with smaller computational complexity and a better understanding of the physical meaning. ${ }^{15-17}$ In this paper, the convergence properties of the time-domain MEFX LMS algorithm for a multiple noise sources and multiple control point ANC system are analysed in the frequency domain. The analysis method presented facilitates a better understanding of the physical meaning, such as the effects of multiple secondary paths (secondary path matrix), and of the correlations among the output of the reference sensors on the convergence speed of the MEFX LMS algorithm. Finally, the results of computer simulations are presented in order to verify the derived theory.

\section{A MULTIPLE NOISE SOURCE AND MULTIPLE CONTROL POINT ANC SYSTEM}

Figure 1 shows a general ANC system for multiple noise sources and multiple control points. In Fig. 1, $I$ is the number of noise sources, $K$ is the number of reference sensors of the adaptive digital filter (ADF) array, composed of finite impulse response (FIR) filters, $M$ is the number of secondary sources, and $L$ is the number of control points (error sensors). Such a system will be referred to as CASE $[I, K, M, L]$ in this paper. There are $M \times L$ different secondary paths (secondary path matrix) between each secondary source and each error sensor, each of which has to be modelled and used $K$ times to generate the array of the $K \times M$ adaptive filters in the controller, which drive every secondary source excited by every reference signal. In this paper, the multiple secondary paths are assumed to be time invariant.

\subsection{The Adaptive Algorithm in Time Domain}

As is shown in Fig. 1, the adaptive controller generates the outputs from $M$ secondary sources, so that the sum of the powers of $L$ error sensors is minimised. This application has led to the development of the MEFX LMS algorithm, which is an extension of the filtered-x LMS algorithm for the CASE $[I, K, M, L]$ ANC system. 